\title{
Algunas Reflexiones Sobre el Cálculo de la Distancia Referencial
}

(Some Thoughts on the Calculation of the Referential Distance)

Victoria VÁzQuez Rozas

(Universidade de Santiago de Compostela)

ABSTRACT: The measures of referential distance proposed in Givon (1983) are still to this date the only independent method to quantify textually the degree of accessibility of the discourse entities. Taking into account the ideas presented in Givon (1992), in the paper a new attempt to compute the referential distance of definite NPs is uphold. This new proposal will allow to overcome the difficulties raised by the bid of Givon (1983), which was applied to Spanish by Bentivoglio (1983). The analysis, based on a corpus of Spanish newspaper texts, leads us to the necessity of distinguishing between anaphoric NPs and non-anaphoric NPs when applying the computation, and shows the suitability of a new model of syntactic subcategorization of referential devices.

KEY-WORDS: Topic continuity; Referential distance; Accessibility; Anaphora.

Resumo: Os cômputos de distância referencial propostos por Givón (1983) constituem, até a data, o unico método independente para a quantificação textual do grau de acessibilidade das entidades do discurso. Levando em consideração as posteriores reflexões de Givón (1992), elabora-se uma proposta alternativa para a estimação da distância referencial das frases nominais definidas, o que permite superar os problemas que projetava a formulação inicial de Givón (1983), aplicada ao espanbol por Bentivoglio (1983). A nossa análise, que está baseada num corpus de textos jornalísticos do espanhol contemporâneo, por um lado, leva-nos à conclusão de que é preciso estabelecer a distinção entre frases nominaiss anafóricas e frases nominais não anafóricas ao aplicar os cômputos, e por outro lado, sugere-nos a conveniência de um novo modelo de subcategorização sintática dos procedimentos referenciais.

Palavras-chave: Continuidade temática; Distância referencial; Acessibilidade; Anáfora.

D.E.L.T.A., 20:1, $2004(27-47)$ 


\section{Introducción}

Reciben el nombre de expresiones referenciales aquellas unidades lingüísticas que cumplen la función básica en la construcción del discurso consistente en la identificación de las entidades sobre las que queremos hablar.

Si la identificación de una entidad implica una primera mención del referente en cuestión, estaremos ante la introducción de un nuevo elemento en el universo de discurso, como ocurre con la secuencia en cursiva de (1):

(1) "Una dotación de la Guardia Civil con perros logró localizar a la anciana (...)" (2voz 28,3,2,1)

Pero también puede suceder que la entidad referida haya sido introducida previamente, de tal forma que la nueva mención supone el mantenimiento o seguimiento de un referente ya establecido, una función que realiza el clítico le en (2) con respecto a la mención anterior Mariano Rajoy, y el demostrativo éste con respecto al nombre Morris Frank en (3) ${ }^{1}$ :

(2) “El joven abordó a Mariano Rajoy en una discoteca pontevedresa, y le aseguró que estaba encargado de su protección” (1voz 25,2,1,25)

(3) "En octubre de 1989 Morris Franklin se inició en esta técnica en Estados Unidos, con Schuesler. Éste la ha utilizado hasta ahora con 140 enfermos" (1voz $67,1,2,6)$

A la vista de los ejemplos anteriores, cabría pensar en una especialización de los distintos tipos de expresiones referenciales, según la cual las frases nominales indefinidas - como en (1) - constituirían el medio idóneo para la introducción de referentes nuevos, mientras que los pronombres - como en (2) y (3) - serían indicadores de continuidad referencial.

Sin embargo, el funcionamiento de las expresiones referenciales en el discurso se revela como un fenómeno bastante más complejo de lo que sugieren los ejemplos citados. Por una parte, la introducción de referentes nuevos no es patrimonio exclusivo de las frases nominales indefinidas,

1 El fenómeno del seguimiento referencial (reference tracking) es analizado, por ejemplo, en Du Bois (1980) y Comrie (1998). 
según se comprueba en (4) y (5), ejemplos en los que se han marcado en cursiva unidades que constituyen primeras menciones en el discurso:

(4) "[el suceso] ha suscitado preocupación entre los universitarios de Santiago" (2vOz $28,4,1,2)$

(5) “Detenido un perturbado que ofreció su 'protección' a Mariano Rajoy" (1voz $252,0,2)$

Por otro lado, si bien atribuimos a los pronombres el estatus de 'información dada' (cf. Halliday 1967:207 y ss.), su funcionamiento como identificadores referenciales no lleva aparejada necesariamente una mención textual previa de la entidad referida. Como se puede ver en el ejemplo (6), el pronombre lo no requiere una referencia textual previa al elemento mencionado:

(6) ten un anuncio gráfico de licort "Tómelo con bielo"

Además, la expresión de la continuidad referencial no es una prerrogativa de los pronombres, sino que puede manifestarse a través de frases nominales definidas y nombres propios, recursos que, como acabamos de ver en (4) y (5), sirven también para la introducción de entidades nuevas en el discurso. En (7) un nombre propio, Orlando C., reintroduce un elemento citado previamente de forma similar. En (8), el letrado y el sacerdote remiten igualmente a entidades introducidas con anterioridad:

(7) "El exdirector de la sucursal del Banco Urquijo en Orense, Orlando C.M. ha sido condenado [...]. La sentencia declara probado que Orlando C. se hizo cargo en 1985 de la dirección de la mencionada oficina” (2voz 28,1,1,18)

(8) “El sacerdote, Josep I.S.B , [... ] no tiene intención de volver a España [...] según indicó su abogado, Manuel B. [...] El letrado indicó que el sacerdote se puso en contacto telefónico con él [...]” (1voz 20,2,1,11)

Aunque los ejemplos anteriores podrían sugerir una relación aleatoria entre función discursiva y tipo de expresión referencial, un examen más atento del fenómeno revelará que la selección del tipo de procedimiento referencial está condicionada por factores discursivos. 


\section{La teoría de la accesibilidad referencial ${ }^{2}$}

Buena parte de las teorías que pretenden dar cuenta del funcionamiento discursivo de los mecanismos referenciales constatan la necesidad de establecer distinciones más refinadas que la simple dicotomía 'entidad nueva en el discurso' vs. 'entidad previamente introducida en el discurso'.

En esta línea tuvo especial repercusión la propuesta de Prince (1981), que configura una escala con siete valores distintos de lo que denomina "Assumed Familiarity". Debemos tener en cuenta, no obstante, que la clasificación de Prince no incorpora un modelo de las correspondencias entre los diferentes estatus cognitivos de las entidades y sus formas sintácticas, pues las clases que propone no son clases lingüísticas sino propiedades de los referentes del discurso ${ }^{3}$. Aun así, la aportación de Prince constituye un punto de referencia para propuestas posteriores en las que se defienden correlaciones específicas entre formas sintácticas y estatus cognitivos de las entidades del discurso y supone, por tanto, un punto de referencia ineludible para la configuración de la llamada teoría de la accesibilidad referencial.

En la misma línea hay que subrayar la importancia de las investigaciones desarrolladas en el marco de la "Hipótesis de la continuidad de tópico (o hipótesis de la continuidad temática o referencial), original de T. (cf. Givón 1983).

La hipótesis de la continuidad de tópico sostiene que los distintos mecanismos gramaticales para la codificación de las entidades a las que se hace referencia en el discurso se pueden situar a lo largo de una escala correspondiente al grado de «accesibilidad» o «disponibilidad» de dichos referentes:

2 Dados los objetivos del trabajo, dejamos en un segundo plano las divergencias existentes entre los distintos modelos que englobamos en la 'Teoría de la Accesibilidad' (cf. Apothéloz 1995: 48-52). Para una comparación entre las diversas propuestas, vid. Huang (2000); para una visión panorámica sobre la teoría vid. Ariel (2001).

3 Un problema distinto es la aplicación de las distinciones propuestas por Prince (1981) al análisis de los usos discursivos concretos. De las dificultades de tal tarea da buena cuenta Poesio y Vieira (1998). 


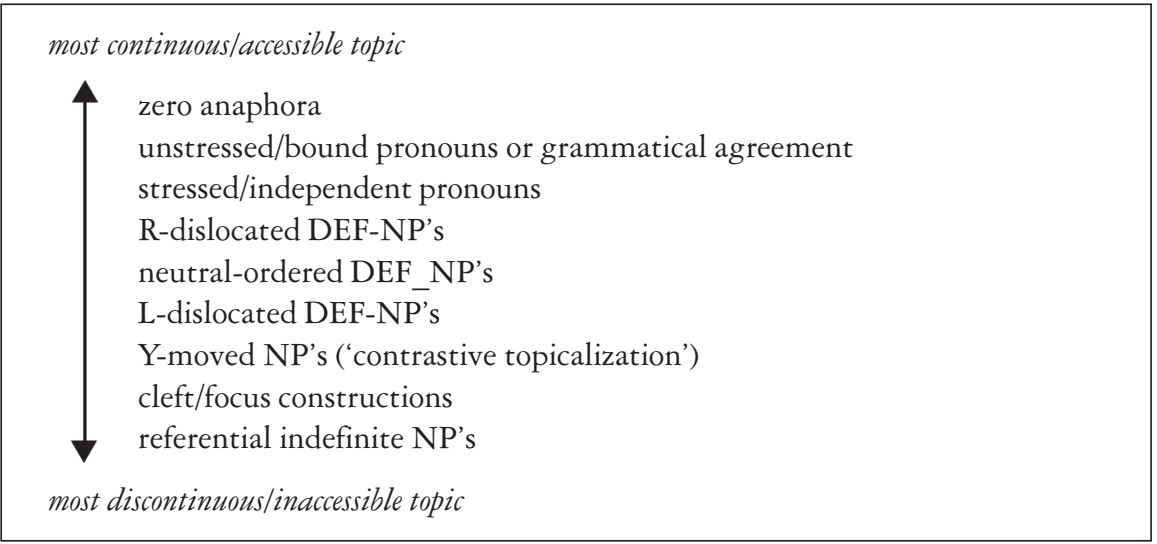

Figura 1 - Escala de Continuidad de Tópico (Givón 1983:17)

Se considera que un referente es más accesible si, en un determinado punto del discurso, el hablante supone que está 'disponible' en la conciencia de su interlocutor. El grado de activación cognitiva de las entidades en la mente de los hablantes puede ir desde un máximo nivel de disponibilidad para las entidades contextualmente muy accesibles, a un nivel mínimo o de no activación para aquellas otras totalmente nuevas en el contexto.

Por lo que se refiere a la expresión sintáctica de los diferentes grados de activación, Givón observa que la accesibilidad se manifiesta en la codificación lingüística de los referentes ("topics") siguiendo un principio de iconicidad:

"The more disruptive, surprising, discontinuous or hard to process a topic is, the more coding material must be assigned to it”. (Givón 1983:18).

Principio que podríamos formular también en términos de cantidad de contenido descriptivo: cuanto más accesible sea un referente, menos contenido descriptivo será necesario para su identificación, y por el contrario, cuanto menos accesible sea, más contenido descriptivo poseerá la expresión seleccionada. Es decir, la accesibilidad alta se correlaciona con el empleo de recursos anafóricos débiles, como la concordancia o los clíticos, mientras que la accesibilidad baja implica el uso de frases nominales léxicamente plenas. 
Diversos autores han desarrollado análisis que podemos enmarcar dentro de la teoría de la accesibilidad (cf. Ariel 1990; Gundel et al. 1993; Chafe 1996). En estos trabajos se nos ofrecen diversas propuestas de ordenación de las expresiones referenciales en función de su nivel de accesibilidad. Un ejemplo que destacamos aquí por su grado de especificidad es la escala de marcación de accesibilidad diseñada por Ariel a partir de los datos distribucionales de diversas lenguas:

Low accessibility

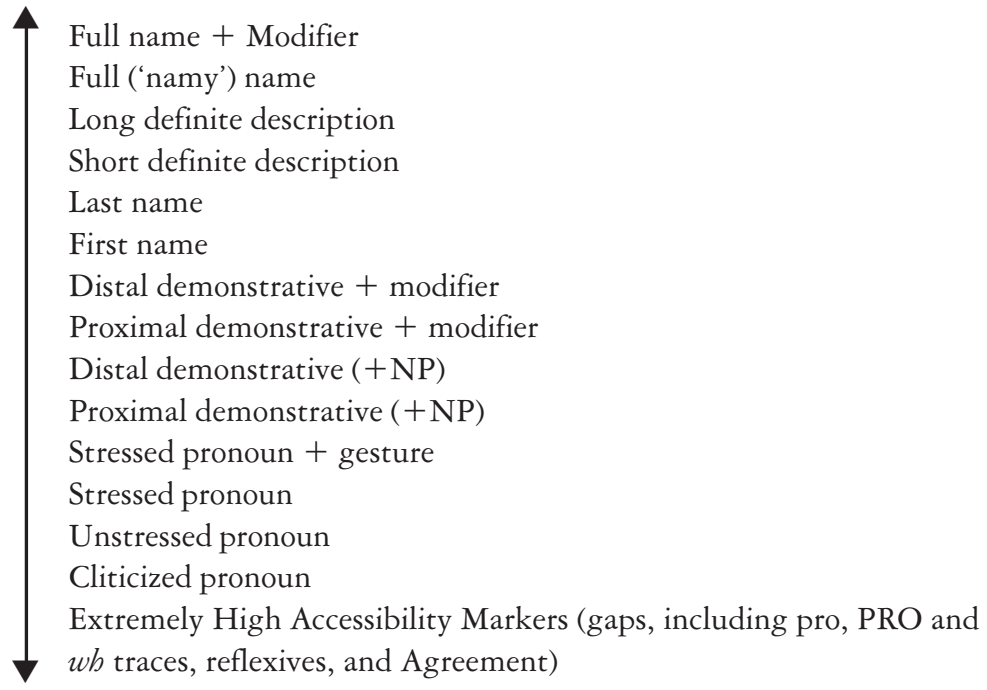

High accessibility

Figura 2 - Escala de Marcación de Accesibilidad (Ariel 1990:73)

Para resumir en pocas palabras la idea básica de la teoría de la accesibilidad, podemos decir que cada expresión referencial constituye una instrucción al receptor para recuperar de la memoria una cierta unidad de información dada, pues el tipo de expresión indica el grado de accesibilidad de la unidad referida en un momento dado del intercambio comunicativo. Las expresiones referenciales son, por tanto, indicativas del estatus cognitivo de los referentes, o más exactamente, de las asunciones del emisor sobre la representación mental del referente que tiene el receptor. 
El análisis que se deriva de la teoría de la accesibilidad es, sin duda, intuitivamente plausible, y encaja perfectamente en el principio de iconicidad mencionado según el cual a menor coste cognitivo - mayor accesibilidad -, menor carga de material léxico será necesaria (de ahí el empleo de desinencias y clíticos), mientras que un mayor coste cognitivo de activación - menor accesibilidad - requerirá el empleo de expresiones léxicamente plenas (frases nominales). No obstante, estamos ante una hipótesis que necesita demostración, pues el estatus cognitivo de las entidades en la mente de los hablantes (es decir, su grado de activación o accesibilidad) no es un dato directamente observable, ya que no podemos penetrar en la mente de los interlocutores para determinar si un concepto está activado o no.

Así pues, para evitar argumentaciones circulares, hay que recurrir a algún procedimiento independiente que permita determinar el estatus de accesibilidad de los referentes. Dado que dicho estatus es una propiedad de carácter conceptual, sólo podremos acceder a ella a través de indicios indirectos, que debemos extraer necesariamente del contexto discursivo.

El contexto está configurado por tres componentes (cf. Givón 1992:12):

a) El contexto situacional o situación concreta de comunicación en que tiene lugar el intercambio comunicativo; este componente juega un papel primordial en la identificación referencial de los elementos deícticos, como ocurre con el demostrativo esto y el pronombre vosotras en el siguiente fragmento conversacional:

(9) M:[de todas] formas os dejo esto§

G:

$\S$ ah / pues [sí]

F:

[ah] pues sí / muchas gracias

G: [vale]

M: [nada] / a vosotras $\S$

b) El contexto cultural compartido o conocimiento enciclopédico común a los interlocutores, que interviene por ejemplo en la identificación referencial de frases nominales definidas y nombres propios usados como primeras menciones (cf. supra ejemplos 4 y 5).

c) El contexto discursivo previo o contexto lingüístico, que ha recibido también la denominación de co-texto. 
Tanto el contexto situacional como el contexto cultural se resisten, por su alto grado de variabilidad, a un análisis cuantitativo que permita obtener datos contrastables, y por tanto sólo el contexto lingüístico proporciona una base empírica de la que se pueden obtener indicios indirectos, pero computables, acerca del funcionamiento discursivo de las expresiones referenciales.

Según señala Givón (1983:113) el objetivo es establecer correlaciones entre el procedimiento referencial empleado en cada caso y ciertos parámetros textuales cuantificables que se interpretan como índices del grado de activación de la entidad correspondiente. En el siguiente apartado nos centraremos en el análisis de la aplicación que se ha hecho al español de uno de estos parámetros, el de la "distancia referencial".

\section{E1 'modelo de la distancia'}

Uno de los métodos cuantitativos empleados en el marco de la teoría de la accesibilidad consiste en la asignación a cada expresión referencial de un índice numérico que da cuenta de la distancia textual, medida en número de cláusulas interpuestas, entre dicha expresión y la anterior mención del mismo referente en el texto. La distancia textual o "distancia referencial" se interpreta, pues, como una medida inversamente proporcional de la accesibilidad referencial de las entidades del discurso: a menor distancia referencial, mayor accesibilidad, y a mayor distancia referencial, menor accesibilidad.

En el texto (10) vemos, por ejemplo, que la distancia referencial de la expresión este es de 1, puesto que su antecedente (su interlocutor) está en la cláusula inmediatamente anterior. Su accesibilidad referencial es, por tanto, mayor que la del referente de el presidente soviético, cuyo antecedente (éste) está a 3 cláusulas de distancia:

(10) "El presidente de la Unión Soviética, Mijail Gorbachov, se entrevistó ayer en la Embajada soviética en Madrid con el primer ministro de Israel, Isaac Shamir. El jefe del Gobierno de Tel Aviv invitó a su interlocutor ${ }_{i}$ a visitar Israel y éste ${ }_{i j}$ no descartó en absoluto la realización de un viaje oficial. Sin embargo, no se ha fijado fecha ni se ha entrado en detalles al respecto. El presidente soviético ${ }_{j}$ condenó duramente «el trágico atentado en los territorios ocupados» en el que murieran anteanoche dos personas y cinco resultaran heridas". (1 voz 3,2,1-2) 
Los estudios llevados a cabo sobre diversas lenguas parecen confirmar la pertinencia del valor distancia para configurar una escala de accesibilidad de los distintos tipos sintácticos de expresión referencial. Por lo que se refiere al español contamos con los datos de Bentivoglio (1983), que aplica la propuesta de análisis cuantitativo de Givón (1983) a un corpus de lengua oral correspondiente al español de Caracas, de Ciudad de México y de Santiago de Chile ${ }^{4}$.

En Bentivoglio (1983) se nos presenta una aplicación al español de lo que podemos llamar el 'modelo de la distancia', que establece diferentes niveles de accesibilidad referencial en función de las medias de distancia referencial de cada tipo de unidad. Sin embargo, observamos que tanto el modelo mismo como la aplicación concreta que de él hace Bentivoglio presentan algunos aspectos problemáticos que es necesario comentar. En primer lugar (apdo. 3.1.) justificaremos la necesidad de prescindir de las expresiones no anafóricas en los cómputos de la distancia referencial; a continuación (apdo. 3.2.) observaremos que ciertas deficiencias del modelo pueden ser corregidas mediante una propuesta más adecuada de clasificación de las expresiones referenciales. Finalmente, en el apdo 4. formularemos una propuesta alternativa de cuantificación de la distancia referencial referida a la categoría de las frases nominales determinadas

\subsection{Expresiones referenciales anafóricas y no anafóricas}

Teniendo en cuenta que la accesibilidad de un referente no procede únicamente de que haya sido (o no haya sido) mencionado explícitamente en el contexto lingüístico previo, sino que puede venir dada también por la situación en la que se produce el intercambio comunicativo (referencia deíctica) y por el contexto cultural compartido, los cómputos de distancia referencial deberían aplicarse únicamente a aquellas expresiones que presentan un antecedente textual, es decir a las expresiones anafóricas. Sin embargo, el método propuesto por Givón y aplicado al español por

\footnotetext{
4 Debemos mencionar aquí también el trabajo de Fant (1985), pues en él se defiende una hipótesis compatible con la propuesta de Bentivoglio (1983) ya que en ambos casos se ponen en relación valores pragmático-discursivos con procedimientos de codificación sintáctica utilizando métodos cuantitativos. Los resultados obtenidos por Fant a partir de una muestra de 3.185 palabras de El habla culta de la ciudad de Madrid muestran que los valores medios de distancia son, como era de esperar, mayores en el caso de las frases nominales que en el caso de los clíticos.
} 
Bentivoglio asigna un valor máximo de distancia referencial (20) a todas las expresiones que no poseen antecedente textual. De esta forma se prejuzga su función discursiva, al atribuirles arbitrariamente una accesibilidad mínima que no queda en absoluto demostrada.

Una muestra de lo inadecuado de tal decisión nos viene servida por el ejemplo que aduce la propia Bentivoglio al explicar su aplicación del cómputo de la distancia referencial. A propósito de la secuencia (11)

(11) "a. ...mi abuelo era hijo de ese tipo que no quería que sus hijos aprendieran a leer",

afirma que

"Mi abuelo in (a) is mentioned for the first time in the discourse as a definite NP. Since this topic is not mentioned in prior clauses, the value assigned for the parameter DISTANCE is de maximum established of 20" (Bentivoglio 1983:260)

Sin embargo en este caso el grado de accesibilidad de la entidad no deriva de una mención previa (que no existe) sino de su relación, marcada textualmente por el posesivo de primera persona $m i$, con otra entidad del discurso, el hablante, que sí presenta alta accesibilidad (por el contexto de situación, que incluye entre los elementos "dados" a las personas del discurso, el emisor y el receptor). En términos de Prince (1981) diríamos que el referente de mi abuelo es un "inferible”, pues su 'familiaridad' procede de su relación con otro referente "evocado en la situación” (el yo hablante); sin embargo, su accesibilidad - relativamente alta - se penaliza con un índice alto de distancia referencial por proceder del contexto deíctico o situacional y no del contexto lingüístico.

Otro tanto sucede con las expresiones referenciales que denotan entidades accesibles en virtud del contexto cultural compartido por los interlocutores, como ocurre con la mayor parte de los usos de expresiones como el sol, el presidente y similares (cf. Givón 1992:13), que no obstante recibirían un valor 20 de distancia (i.e., mínima accesibilidad) en una primera mención textual.

La aplicación del parámetro distancia en los términos que estamos comentando nos obliga a asignar el mismo valor de accesibilidad a expresiones 
tan dispares como por ejemplo la citada en (11) y las siguientes (interpretando los segmentos en cursiva como primeras menciones):

(12) El próximo domingo iremos a comer con el abuelo

(13) Le da clase un profesor italiano

(14) No puedo ir al concierto porque he perdido la entrada

El ejemplo (14) nos lleva a comentar otro aspecto del método de cuantificación de la distancia referencial que va en contra de la correlación 'a mayor distancia, menor accesibilidad,'. Se trata de la exigencia de correferencia entre la expresión referencial y su antecedente textual, que no se cuestiona en la aplicación de Bentivoglio. No hay lugar, pues, para las "anáforas asociativas" o "anáforas indirectas", que resultan ser una fuente relevante de accesibilidad para las frases nominales definidas ${ }^{5}$.

\subsection{Tipología de expresiones referenciales}

El modelo de la distancia prevé que una vez asignado el índice de distancia referencial a cada expresión referencial es necesario calcular las medias de distancia referencial para cada uno de los tipos morfosintácticos de expresión referencial. Estas medias definirán la escala o jerarquía de procedimientos referenciales correlativa a la escala de accesibilidad cognitiva.

Sin embargo, el manejo aislado de los valores medios de distancia referencial oculta el funcionamiento discursivo real de las expresiones

\footnotetext{
5 En Givón (1992:13) se reconocen, aunque no con el término de "anáfora asociativa o indirecta", las características peculiares de este tipo de expresiones referenciales, cuya accesibilidad resulta de la conjunción de la información proveniente del contexto lingüístico (antecedente lingüístico no correferencial) con los datos del contexto cultural. A propósito de los ejemplos (10b) y (10c)

(10) b. They showed us this gorgeous house, but the living room was too small

c. She went into a restaurant and asked to see the menu...,

observa Givón:

"the accessibility of 'the living room' in (10b) is due in part to the presence of 'this gorgeous house' in the immediately preceding text, but is part also to the shared cultural knowledge that 'a prototypical house has one living room'. And the accessibility of 'the menu' in (10c) is due in part to the presence of 'a restaurant' in the immediately preceding text, but in part also to the shared cultural knowledge - frame, schema, script - that 'a typical restaurant has a menu'." (Givón 1992:13)
} 
referenciales. Las dificultades surgen especialmente cuando el valor medio de distancia referencial de una cierta categoría sintáctica no caracteriza un porcentaje elevado de los ejemplares de dicha categoría. Es decir, el valor medio de distancia referencial no puede considerarse característico de un tipo de expresión referencial si el grado de dispersión es alto.

En Givón (1992) se reconoce que el 'modelo de la distancia' resulta inadecuado para aquellas clases de distribución dispersa, es decir, las clases (e) y (f) de la siguiente tabla, en la que se comparan los valores medios de distancia referencia (RD, "referential distance")) con el grado de distribución categorial de los procedimientos de codificación referencial más comunes ${ }^{6}$ :

\begin{tabular}{lcl}
\hline construction & $\begin{array}{c}\text { mean } R D \\
\text { (no.of clauses) }\end{array}$ & $\begin{array}{l}\text { degree of categorial } \\
\text { distribution }(\%)\end{array}$ \\
\hline a. zero anaphora & 1.0 & $100 \%$ at mean \\
b. unstressed PRO & 1.0 & $95 \%$ at mean \\
c. stressed PRO & 2.5 & $90 \%$ between $2-3$ \\
d. Y movement & 2.5 & $90 \%$ between $2-3$ \\
e. DEF noun & 7.0 & $25 \%$ at 1.0 \\
& & $35 \%$ scattered $5.0-19$ \\
& & $40 \%$ at $20+$ \\
f. modified DEF_NP & 10.0 & $55 \%$ scattered 5.0-19 \\
& & $45 \%$ at $20+$ \\
g. L-dislocated DEF-NP & 15.0 & $60 \%$ at $20+$ \\
& & $(25 \%$ at $4-9)$ \\
& & $(13 \%$ at $10-19)$ \\
h. repeated DEF-NP & $75 \%$ at $20+$ \\
& 17.0 & $(18 \%$ at 3-8) \\
& & $(6 \%$ at $15-19)$ \\
\hline
\end{tabular}

Tabla 1 - Distribución Categorial de los Índices de Distancia Referencial (Givón 1992:21)

6 El comentario que hace Givón de los datos de la tabla es el que sigue:

"Taking first the first four (a)-(d) and the last two (g) and (h) devices, their populations' distribution is either categorial or approaching categoriality. In contrast, the two mid-distance devices ? definite noun (e) and restrictively modified definite NP (f) ? show a scattered, noncategorial distribution. Further, nearly half of their membership has no anaphoric antecedence at all within the preceding 20 clauses. No SPECIFIC RD value characterizes these grammatical devices. Our RD measure is relatively useless for describing them" (Givón 1992: 21) 
Efectivamente, por ejemplo la clase (e) de los 'nombres con determinante definido' ("DEF noun”) presenta índices de distancia referencial muy poco homogéneos. Así, la expresión referencial mi abuelo de (11) muestra una distancia referencial de 20, pues carece de antecedente textual, mientras que una expresión referencial similar como su interlocutor en (10) presenta una distancia referencial de 1 , pues el mismo referente es mencionado en la cláusula inmediatamente anterior.

Si la medida de distancia referencial resulta inútil para describir la función discursiva de las categorías 'nombre con determinante definido' y 'frase nominal determinada modificada', ello se debe, por una parte, a lo señalado en el apartado 3.1, es decir a la inclusión en los cómputos de las expresiones referenciales no anafóricas, y por otra parte, a las deficiencias inherentes a la tipología de categorías morfosintácticas manejada. Las clases categoriales que distingue Bentivoglio (1983) son las siguientes:

1. Concordancia verbal y pronombres átonos (personales y posesivos)

2. Pronombres tónicos (personales y demostrativos)

3. Pronombres tónicos con clíticos correferenciales

4. Frases nominales con clíticos correferenciales

5. Frases nominales definidas:

a) Sólo con artículo definido

b) Con determinante demostrativo, posesivo; con modificador "genitivo" o adjetivo.

c) Con modificador cláusula relativa.

6. Nombres propios

7. Frases nominales en construcciones existenciales y presentativas.

a) Definidas.

b) Indefinidas

La tipología es, en apariencia, bastante detallada. Sin embargo, a poco que se examinen las clases propuestas surgen las objeciones. En general, sería mucho más útil una clasificación que permitiese ofrecer cómputos 
diferenciados para categorías claramente distintas, o de cuyo funcionamiento homogéneo en el discurso no tengamos pruebas concluyentes, como ocurre por ejemplo con los pronombres tónicos personales y demostrativos.

Por otra parte, el criterio que se sigue para agrupar pronombres tónicos personales y demostrativos en (2) no se mantiene en (5) (supondría la agrupación de frase nominal con artículo y frase nominal con determinante demostrativo), sino que, por alguna razón que no se explicita, se propone ahora una distinción entre las subclases (5) a-b-c que privilegia arbitrariamente ciertas configuraciones funcionales - las frases constituidas por artículo y sustantivo (5a), y las frases con relativas en función de modificador (5c) -, y sitúa en un segundo plano otros tipos de constituyentes frásicos, que se reúnen en el 'cajón de sastre' de la clase (5b) ${ }^{7}$.

Los ejemplos con los que ilustra Bentivoglio la clase (5b) son: "esta señora", "su marido", "el marido de la señora", "la pobre señora", "el nuevo aparato ese" y "sus estudios superiores" (cf. Bentivoglio 1983:275-276). Y aunque la propia autora reconoce la conveniencia de separar los tipos de frase representados por cada uno de los ejemplos citados ${ }^{8}$, los cómputos que ofrece en su estudio tienen carácter global.

Como indicio de la diferente función discursiva de algunas de las categorías reunidas en (5b), basta pensar en que una frase nominal con determinante artículo o posesivo puede constituir una primera mención de la entidad en el discurso ("su marido", "el marido de la señora”), mientras que las frases nominales demostrativas suponen normalmente una relación anafórica (cf. Maes y Noordman 1995: 258).

Si por un lado no se justifica la pertinencia de ciertas subclases ( $5 \mathrm{a}-\mathrm{b}-\mathrm{c})$, por otro se echa de menos una mayor compartimentación de los datos que permitiría comprobar diferencias de accesibilidad entre subtipos de expresiones referenciales. En el siguiente apartado me referiré en particular a la distinción entre modificadores restrictivos y no restrictivos, que reclama

\footnotetext{
Téngase en cuenta, además, que la clase $(5 \mathrm{c})$ agrupa todas las frases nominales definidas con una cláusula relativa como modificador, independientemente del tipo de determinante que posean (artículo, demostrativo o posesivo) y de la presencia de otros modificadores (adjetivos, frases preposicionales...).

8 "It is clear that in a more extended analysis it would be advisable to examine separately all types of M-NP's, as they show different degrees in regard to continuity" (Bentivoglio 1983:276).
} 
un análisis más detallado. Hay razones para pensar que las frases con modificadores no restrictivos remiten a entidades más accesibles que las representadas por frases con modificadores restrictivos (cf. Bolinger 1972 y López García 1998:183).

\section{Propuesta alternativa para el cómputo de la distancia referencial de las frases nominales determinadas}

Según se desprende de la exposición precedente, las deficiencias más notorias del modelo de la distancia atañen a la caracterización de las frases nominales determinadas. Lo que me propongo mostrar a continuación es que tales deficiencias no provienen en sí de la teoría de la continuidad temática, sino que resultan de una aplicación incorrecta de sus postulados? ${ }^{9}$. Si corregimos dichos errores, quizá lleguemos a la conclusión de que el modelo resulta operativo también en el caso de las frases nominales determinadas.

En primer lugar, hay que prestar especial atención a la selección del corpus que será objeto de análisis. No debemos perder de vista que los índices de distancia referencial constituyen una medida de la accesibilidad textual - lingüística - de los referentes discursivos, pero no nos dicen nada acerca de la accesibilidad proveniente de la situación o del contexto cultural compartido. Por consiguiente, nuestra selección de textos ha de minimizar en la medida de lo posible la accesibilidad derivada del contexto situacional y cultural ${ }^{10}$.

En nuestro caso el corpus sobre el que haremos los cómputos está constituido por 8 textos de periódico (La Voz de Galicia y El País), todos ellos encuadrables en el género 'crónica de sucesos'. Estos textos suman un total de 2.452 palabras.

\footnotetext{
9 La observación no se refiere en particular a la aplicación de Bentivoglio (1983), sino a las líneas generales del modelo que indican cómo se realizan los cómputos de distancia referencial y a cómo se ofrecen los resultados de dichos cómputos.

10 Se ha de procurar un grado de generalidad suficiente para evitar fuentes individuales e idosincrásicas de accesibilidad. En este sentido, cabe esperar que el conocimiento general compartido por emisor y receptor tendrá menor relevancia en las crónicas de sucesos en periódicos que, por ejemplo, en la correspondencia personal entre dos amigos íntimos.
} 
Además, dado que nuestro objetivo es, en este caso, parcial, hemos efectuado los cómputos únicamente sobre un cierto tipo de expresiones referenciales, las frases nominales; y para evitar en la medida de lo posible problemas derivados de la identificación referencial - incluidos los usos no referenciales: inespecíficos, predicativos -, se ha restringido el análisis a las expresiones referenciales de referencia humana. Con estas restricciones, hemos contabilizado 104 frases nominales de referencia humana.

Por lo que se refiere a la aplicación de los cómputos, no se ha asignado valor alguno de distancia referencial a las expresiones referenciales no anafóricas, pues tal asignación distorsiona los datos muy especialmente en la clase de expresiones referenciales estudiadas, que presentan tanto usos anafóricos como no anafóricos. Al no atribuir índice de distancia referencial a las expresiones referenciales sin antecedente textual evitamos prejuzgar su grado de accesibilidad ni la fuente contextual de la que deriva. La tabla siguiente muestra las frecuencias correspondientes a las categorías distinguidas:

\begin{tabular}{ccc}
\hline Fns anafóricas (directas) & Fns anafóricas (indirectas) & Fns no anafóricas \\
\hline 52 & 1 & 51 \\
\hline
\end{tabular}

Tabla 2 - Frecuencia de frases nominales (Fns) anafóricas y no anafóricas

Las frases nominales anafóricas directas presentan un valor de distancia referencial que oscila entre 1 (antecedente en la cláusula inmediatamente anterior) y 16. De los 52 ejemplos, 19 presentan una distancia referencial de 1 y 11 una distancia referencial de 2, lo cual supone unos valores de accesibilidad bastante más altos de lo esperable dado el principio de iconicidad que rige la 'escala de continuidad temática'. En consonancia, la media de distancia referencial es sorprendentemente baja $(3,15)$, y contrasta con los valores que ofrece Bentivoglio para el conjunto de las frases nominales definidas de referente humano: 8,03 para la función sujeto, 7,22 para el 'acusativo', 17,3 para el 'dativo', 12,25 para el 'genitivo' y 11,95 para el 'oblicuo' (cf. Bentivoglio 1983:290).

La diferencia se debe con certeza a la inclusión en los cómputos de Bentivoglio de las expresiones referenciales no anafóricas, a las que, como ya hemos dicho, en el modelo de Givón y Bentivoglio se les asigna 
arbitrariamente un índice 20 de distancia referencial. Para mostrar la repercusión que tal inclusión tiene, hemos calculado la media de distancia referencial del conjunto de las frases nominales determinadas (con un valor 20 de distancia referencial para los 51 casos de frases nominales no anafóricas), y el resultado es 11,49, muy alejado del valor de 3,15 y más en la línea de las medias que ofrece Bentivoglio.

Por otra parte, proponemos una tipología (parcial) de expresiones referenciales en la que no se mezclen clases que muestran un funcionamiento discursivo claramente divergente

Con el objeto de superar las dificultades que observamos en la propuesta de Bentivoglio, y en particular la subclasificación que maneja de las frases nominales definidas, hemos registrado como datos independientes, por un lado, los distintos tipos de determinante $(\varnothing$, indefinido, artículo, posesivo y otros - ambos -), y los distintos tipos de modificadores (frase preposicional, adjetivo/frase adjetiva, relativa, aposición), dejando abierta la posibilidad a todo tipo de combinaciones. Además, se establece la distinción entre modificadores restrictivos y no restrictivos. Dado lo reducido del corpus, que no nos permite avanzar hipótesis consistentes sobre la relevancia de muchas de los rasgos mencionados, me limitaré a señalar la pertinencia de la distinción entre modificadores restrictivos y no restrictivos en relación con el carácter anafórico o no anafórico (primera mención) de las frases nominales definidas.

\begin{tabular}{lcc}
\hline & Fns anafóricas & Fns no anafóricas \\
\hline Con modificador restrictivo & 8 & 16 \\
\hline Sin modificador restrictivo & 44 & 9 \\
\hline TOTAL & 52 & 25 \\
\hline
\end{tabular}

Tabla 3 - Frecuencias de modificación para frases nominales anafóricas y no anafóricas

Si bien los datos de esta tabla resultan por sí mismos bastante reveladores y permiten precisar la relación existente entre la configuración sintáctica de las frases nominales definidas y su función discursiva, no deja de sorprendernos que haya 9 ejemplos de frases nominales no anafóricas sin algún modificador restrictivo. Sin embargo, al comprobar cuáles son las 
expresiones implicadas nuestro inicial desconcierto se desvanece: "La Policía" (2 casos), "La Guardia Civil" (2 casos), "el fiscal", "su abogado, Manuel Bayona”, "su padre, Lisardo Gómez, que trabaja como obrero", "sus agresores". En unos casos la accesibilidad viene dada por el contexto cultural compartido, y en otros por la relación, marcada por el posesivo, con otro referente textual accesible.

\section{Recapitulación y conclusiones}

A la vista de lo expuesto, y en contra de lo apuntado por Givón (1992), creo que el modelo de la distancia, permite dar cuenta del funcionamiento discursivo de las frases nominales definidas (en la misma medida que sirve para los otros tipos de expresiones referenciales). Para ello deben cumplir-se dos condiciones:

a) Es preciso establecer la distinción entre frases nominales anafóricas y frases nominales no anafóricas, pues sólo las primeras deben ser tenidas en cuenta en los cómputos de distancia referencial.

b) Se requiere una subcategorización sintáctica adecuada, que no privilegie arbitrariamente una(s) propiedad(es) frente a otras.

Un asunto diferente, que será necesario abordar, es si los cómputos de distancia referencial son realmente una medida adecuada de la accesibilidad o grado de activación de las entidades del discurso, y, desde una perspectiva teórica más general, si las distintas propuestas englobadas en la Teoría de la Accesibilidad constituyen el marco más adecuado para explicar el función discursiva de las expresiones referenciales.

Por lo que respecta a la primera cuestión, diferentes investigaciones desarrolladas en los últimos años - en bastantes casos relacionadas con la construcción de aplicaciones computacionales - han demostrado que el estatus discursivo-cognitivo de los referentes depende de una serie bastante compleja de factores (cf. Kibrik 1996, Rico Pérez 1996, Mitkov 1996). Incluso si nos limitamos a los mecanismos de activación de referentes provocados por el contexto lingüístico, nos encontramos con que, además de la distancia, entran en juego la estructuración interna del discurso, las propiedades intrínsecas de los referentes (su animación, su protagonismo en el discurso), la función sintáctica, semántica y pragmática de la expresión referencial y del antecedente textual, etc. 
Si tomamos como ejemplo los datos de nuestro corpus, tendremos que reconocer que el modelo de la distancia difícilmente puede explicar que un procedimiento referencial marcado como las frases nominales definidas presente como distribución característica (19 de 52 casos) la distancia referencial 1, es decir, la referencia a una entidad nombrada en la cláusula inmediatamente anterior. Es evidente que otros factores, además de la distancia, resultan relevantes para determinar el grado de accesibilidad de los referentes discursivos.

En cuanto a los presupuestos que fundamentan la Teoría de la Accesibilidad, algunos autores han cuestionado la interpretación de las expresiones referenciales como simples mecanismos para la identificación de los referentes del discurso, interpretación que implica una visión unidimensional de la función discursiva de dichas expresiones. Si la distribución de las expresiones referenciales estuviese guiada únicamente por la función identificadora, no podríamos justificar la selección que de ellas efectúa el emisor en aquellos contextos en los que podrían alternar varios tipos de expresión sin originar dificultades en la identificación de referentes. Por el contrario, si interpretamos las expresiones referenciales como mecanismos polifuncionales, podremos atribuirles, además, otras funciones discursivas.

Diversos estudios llevados a cabo en los últimos años han destacado en particular dos funciones (cf., entre otros, Apothéloz y Reichler-Béguelin 1999):

a) Una función estructuradora del discurso, que relaciona, por ejemplo, el uso de expresiones referenciales marcadas - frases nominales, nombres propios -, frente a los recursos anafóricos débiles - concordancia, clíticos -, con la delimitación de unidades discursivas tales como parágrafos, episodios y eventos. (Cf. Clancy 1980; Fox 1987; Hofmann 1989).

b) Una función de modificación o reclasificación de un referente introducido previamente, función que explicaría, entre otras cosas, el empleo de frases nominales con determinante demostrativo en contextos de mínima distancia referencial (cf. Mederos 1988; Maes y Noordman 1996; Leonetti 1996).

Sin duda el análisis de corpus textuales más amplios que el que hemos manejado aquí permitirá confirmar - o rebatir - la validez de estas propuestas.

E-mail: fevvazq@usc.es Recebido em março de 2003 Aprovado em dezembro de 2003 


\section{REFERENCIAS BibliográficAS}

ApOTHÉLOZ, Dénis. 1995. Rôle et fonctionnement de l'anaphore dans la dynamique textuelle. Xenebra: Droz.

Apothéloz, Dénis \& Reichler-BéGuelin, M.-J. 1999. Interpretations and functions of demonstrative NPs in indirect anaphora. Journal of Pragmatics. 31: 363-397.

Ariel, Mira. 1990. Accessing noun-phrase antecedents. Londres: Routledge. . 2001. Accessibility theory: an overview. In Ted SANDERS, Joost SCHILPEROORD \& Wilbert SpOOREN (Eds.) Text Representation: Linguistic and psycholinguistic aspects. Amsterdam: John Benjamins. 29-87.

Bentivoglio, Paola. 1983. Topic continuity and discontinuity in discourse: A study of spoken latin-american Spanish. In: T. Givón. (Ed.) Topic Continuity in Discourse. A Quantitative Cross-language Study. Amsterdam: Jonh Benjamins: 259-311.

Bolinger, Dwight. 1972. Adjective position again. Hispania, 55: 91-94.

CHAfE, Wallace. 1996. Inferring identifiability and accessibility. In T. Fretheim \& J.K. Gundel (Eds.) Reference and referent accessibility. Amsterdam: John Benjamins: 37-46.

Clancy, Patricia. 1980. Referential choice in English and Japanese narrative discourse. In: Wallace Chafe (Ed.) The Pear Stories. Norwood, Nueva York: Ablex: 127-202.

Comrie, Bernard. 1998. Seguimiento referencial: descripción y explicación. In: Juan de D. Luque Durán \& A. Pamies Beltrán (Eds.) Estudios de Tipología Lingüística. Granada: Método: 37-54

Du Bors, John W. 1980. Beyond Definiteness: The Trace of Identity in Discourse. In: Wallace Chafe (Ed.) The Pear Stories: Cognitive, Cultural, and Linguistic Aspects of Narrative Production. Norwood, New York: Ablex: 204-274.

FANT, Lars. 1985. Procesos anafóricos y valor enfático en español hablado. Español Actual, 43: 5-26.

Fernández Ramírez, Salvador. 1987. Gramática española. 3.2. El pronombre. Madrid:Arco/Libros.

Fox, Barbara. 1987. Discourse structure and anaphora: Written and conversational English. Cambridge: Cambridge University Press.

Givón, T. 1983. Topic continuity in discourse: An introduction. In: T. Givón. (Ed.) Topic Continuity in Discourse. A Quantitative Cross-language Study. Amsterdam: Jonh Benjamins: 5-41. 
- 1992. The grammar of referential coherence as mental processing instructions. Linguistics, 30: 2-55.

Gundel, Jeanette K., Hedberg, Nancy \& Zacharsi, Ron. 1993. Cognitive status and the form of referring expressions. Language, 69/2: 274-307.

Halliday, M.A.K. 1967. Notes on transitivity and theme in English. Part 2. Journal of Linguistics, 2: 199-244.

Hofmann, Thomas R.. 1989. Paragraphs \& anaphora. Journal of Pragmatics, 13: 239-250.

HuANG, Yan. 2000. Discourse anaphora: Four theoretical models. Journal of Pragmatics, 32: 151-176.

KIBRIK, Andrej. 1996. Anaphora in Russian Narrative Prose: A Cognitive Calculative Account. In: Barbara Fox (Ed.) Studies in anaphora. Amsterdam: John Benjamins: 255-303.

Leonetti, Manue. 1996. Determinantes y contenido descriptivo. Español Actual, 66: 5-23.

López García, Ángel. 1998. Gramática del español. III. Las partes de la oración. Madrid: Arco/Libros.

Maes, Alfons A. \& Noordman, Leo G. M. 1995. Demonstrative nominal anaphors: a case of nonidentificational markedness. Linguistics, 33: 255-282.

Mederos Marín, Humberto. 1988. Procedimientos de cohesión en español actual. Tenerife: Excmo. Cabildo Insular de Tenerife.

Mitkov, Ruslan. 1996. Anaphor resolution: a combination of linguistic and statistical approaches. In: S. Botley, J. Glass, T. McEnery \& A. Wilson. (Eds.) Approaches to discourse anaphora (Proceedings of the Discourse Anaphora and Resolution Colloquium, DAARC96). Lancaster: UCREL: 76-85.

Prince, Ellen. 1981. Toward a taxonomy of given-new information. In: O. Cole (Ed.) Radical Pragmatics. New York: Academic Press: 223-255.

Poesio, Maximo \& Vieira, Renata. 1998. A Corpus-based Investigation of Definite Description Use. Computational Linguistics, 24/2: 183-216.

Rico Pérez, Celia. 1994. Estudio de la incidencia de diferentes fuentes de información en el establecimiento de relaciones anafóricas. Boletín de la SEPLN, 14: 63-75. 